\title{
Diameter of surgical versus endoscopic ultrasound-guided gastrojejunostomy: that much wider after all is said and done?
}

Whereas retrospective literature confirms the comparable clinical success of endoscopic ultrasound-guided gastrojejunostomy (EUS-GJ) and surgical gastrojejunostomy (SG]) $[1,2]$, no head-tohead comparison exists of their caliber and long-term patency.

We present one case of a patient receiving both procedures, offering an unusual opportunity for direct comparison ( Video 1).

A 52-year-old patient was diagnosed with gastric outlet obstruction owing to a duodenal B-cell lymphoma. EUS-GJ was performed using a $20-\mathrm{mm}$ lumenapposing metal stent (LAMS), followed by $18-\mathrm{mm}$ balloon dilation [3] ( $>$ Fig. 1). Upon disease remission with chemotherapy, a blind-ended bulb resulted from scarring of the stenotic duodenal tract. Although the EUS-G] was wide and patent ( $\triangleright$ Fig. 2), erosions were appearing on the jejunal side after 8 months ( $>$ Fig. 2 c) and an SGJ was proposed owing to the uncertainty of long-term patency of the EUS-G] and a potentially wider caliber of the SG]. Following surgery, gastrointestinal follow-through (\$Fig. $\mathbf{3}$ ) showed adequate flow through both anastomoses, but EUS-GJ seemed reduced in diameter 13 months after placement. LAMS extraction was planned, revealing significant granulation tissue overgrowth surrounding a stabilized fistula reduced in caliber (> Fig.3).

On that occasion, the SGJ caliber was evaluated. Although the surgeon created an almost 5-cm incision, a 20-mm balloon perfectly fitted the final SGJ ( $\mathbf{F i g . 4 )}$. Indeed, SG] requires a linear incision of stomach and jejunal walls and latero-lateral suturing of their inferior and superior margins [4]. This elliptic anastomosis wil become round after maturation and scarring, with a smaller final circular diameter compared to the initial linear cut ( $>$ Fig. 5)

Pending randomized data, this case suggests a comparable caliber of SG] and

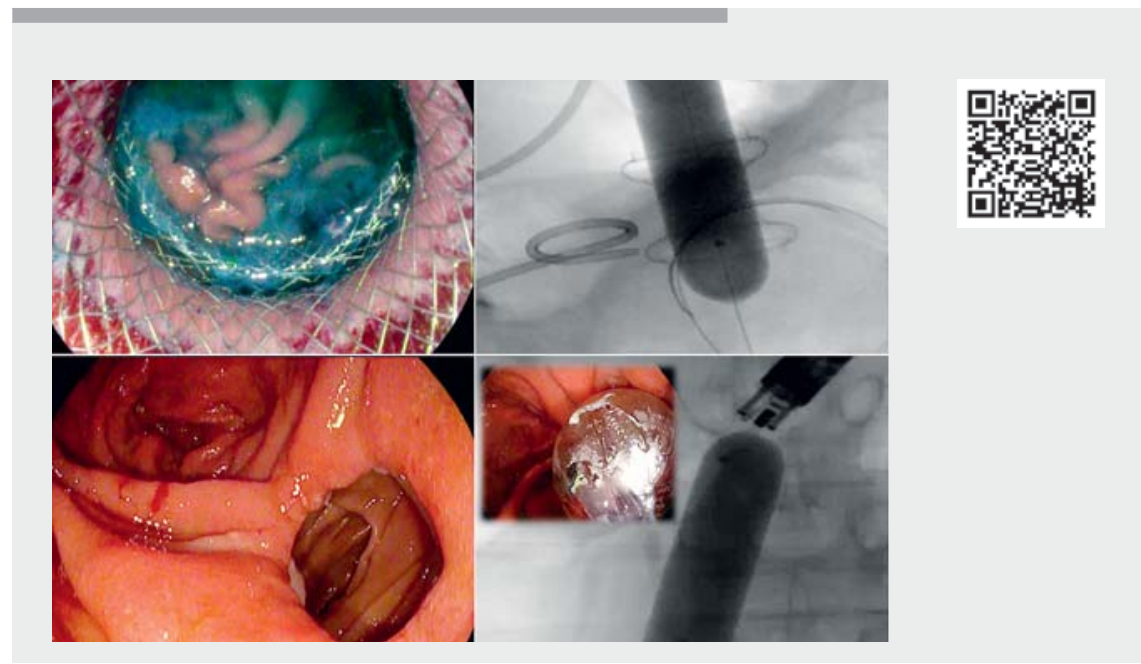

Video 1 A patient receiving both an endoscopic ultrasound-guided and a surgical gastrojejunostomy offered an unusual opportunity to compare the diameter of the 2 anastomoses.
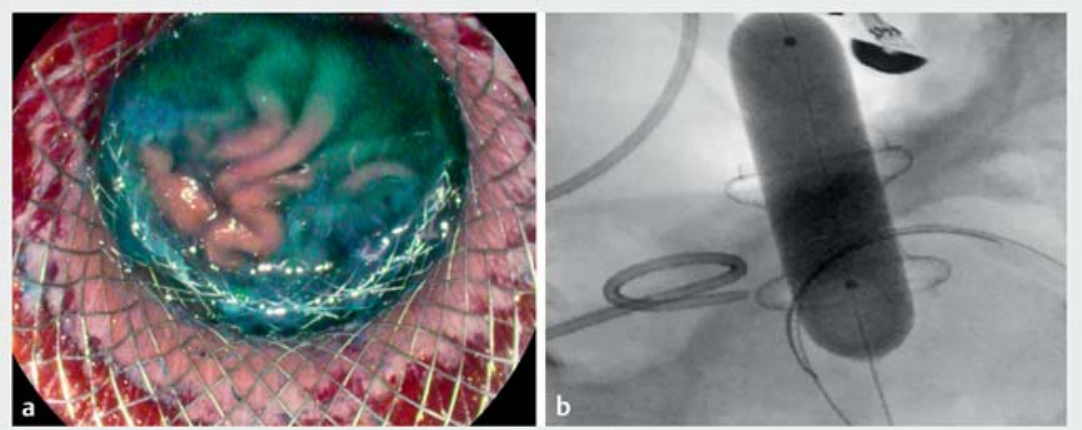

- Fig. 1 Endoscopic ultrasound (EUS)-guided gastrojejunostomy after lumen- apposing metal stent (LAMS) placement. a Endoscopic view of the small intestine through the LAMS, with some blue fluid used for jejunal distention coming into the stomach. $\mathbf{b}$ Radiograph of 18-mm balloon dilating the 20-mm LAMS after placement.

EUS-G] for a substantial part of their history, and therefore an assumed larger diameter should not be used as a reason to prefer SG]. However, it also suggests that long-term LAMS friction may induce inflammatory responses deserving further elucidation, especially when advocating EUS-G] use in benign disease.

Endoscopy_UCTN_Code_TTT_1AT_2AD

\section{Competing interests}

Michiel Bronswijk has consultancy agreements with Prion Medical - Taewoong. Schalk Van der Merwe holds the Cook and Boston-Scientific chair in interventional endoscopy and holds consultancy agreements with Cook, Pentax and Olympus. The remaining authors declare no $\mathrm{COI}$ relevant for this article. 


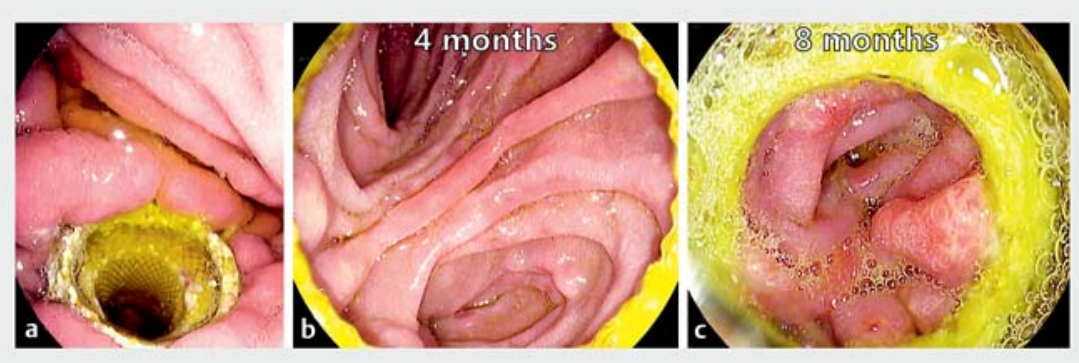

- Fig. 2 Endoscopic follow-up of the endoscopic ultrasound-guided gastrojejunostomy (EUS-GJ). a, b Follow-up at 4 months: wide and patent EUS-G], with normotrophic mucosa on the jejunal side. $c$ Follow-up at 8 months: initial erosions were seen on the jejunal side of the anastomosis.
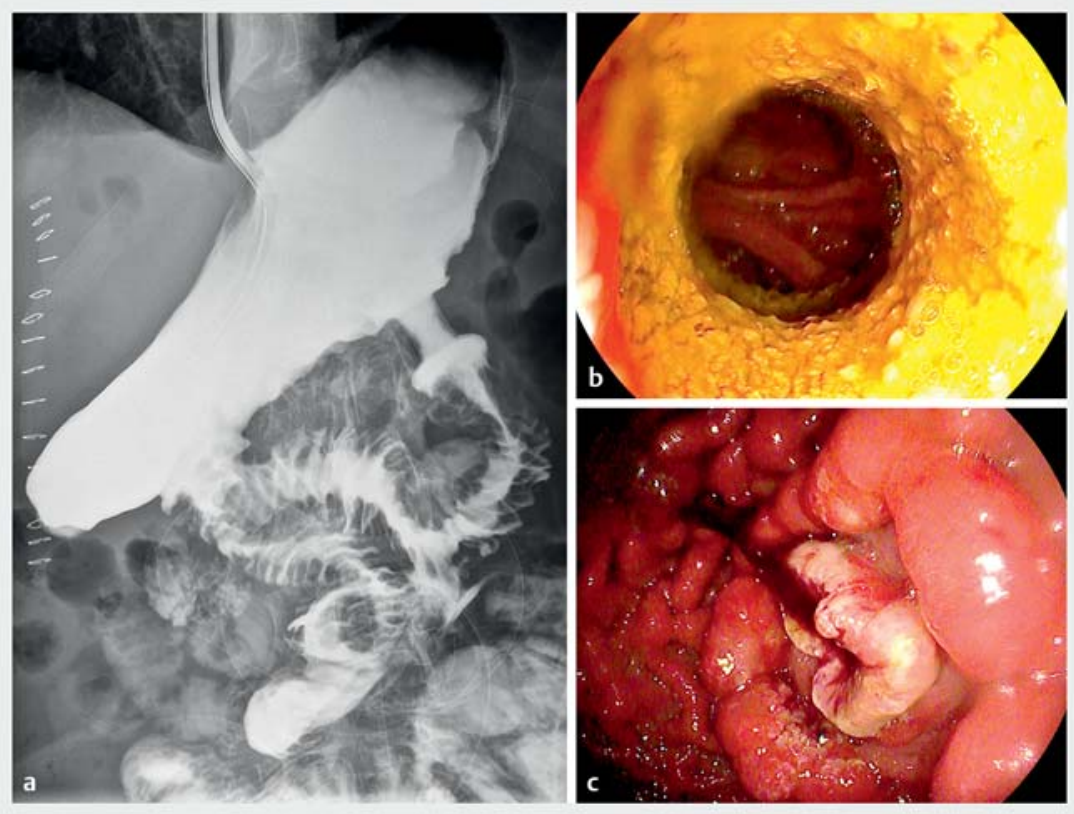

- Fig. 3 a Endoscopic follow-through showing adequate contrast flow through both anastomoses, although EUS-G] diameter seemed slightly reduced after 13 months. b, c Endoscopy performed for lumen-apposing metal stent (LAMS) extraction 13 months after placement. b Endoscopic view of the LAMS reduced in caliber, not passable with a standard gastroscope. c After LAMS removal, significant granulation tissue overgrowth was visible surrounding a stabilized fistula reduced in caliber.
The authors

Giuseppe Vanella ${ }^{1}{ }^{\oplus}$, Domenico Tamburrino² Francesco Vito Mandarino ${ }^{1}$, Michiel Bronswijk $^{3,4}$, Schalk Van der Merwe ${ }^{3}$, Massimo Falconi ${ }^{2}$, Paolo Giorgio Arcidiacono ${ }^{1}$

1 Pancreatobiliary Endoscopy and Endosonography Division, Pancreas Translational \& Clinical Research Center, IRCCS San Raffaele Scientific Institute, Milan, Italy

2 Pancreatic Surgery Unit, Pancreas Translational \& Clinical Research Center, IRCCS San Raffaele Scientific Institute, Milan, Italy

3 Department of Gastroenterology and Hepatology, University Hospitals Gasthuisberg, University of Leuven, Leuven, Belgium.

4 Department of Gastroenterology and Hepatology, Imelda General Hospital, Bonheiden, Belgium

Corresponding author

\section{Giuseppe Vanella, MD}

Pancreatobiliary Endoscopy and

Endosonography Division, Pancreas

Translational \& Clinical Research Center, IRCCS San Raffaele Scientific Institute, VitaSalute San Raffaele University, Via Olgettina 60, 20132, Milan, Italy

Fax: +39-0226435609

vanella.giuseppe@hsr.it

\section{References}

[1] Fan W, Tan S, Wang J et al. Clinical outcomes of endoscopic ultrasound-guided gastroenterostomy for gastric outlet obstruction: a systematic review and meta-analysis. Minim Invasive Ther Allied Technol 2020: 1-9. doi:10.1080/13645706.2020.1792500

[2] Bronswijk M, Vanella G, van Malenstein H et al. Laparoscopic versus EUS-guided gastroenterostomy for gastric outlet obstruction: an international multicenter propensity score-matched comparison (with video). Gastrointest Endosc 2021. doi:10.1016/j. gie.2021.04.006 

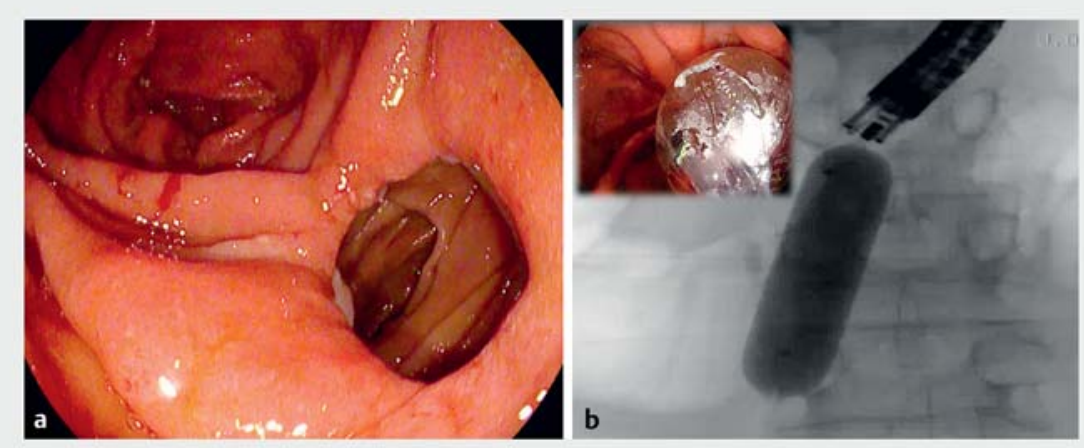

- Fig. 4 Surgical gastrojejunostomy caliber evaluated with a 20-mm dilation balloon, perfectly fitting the anastomosis.

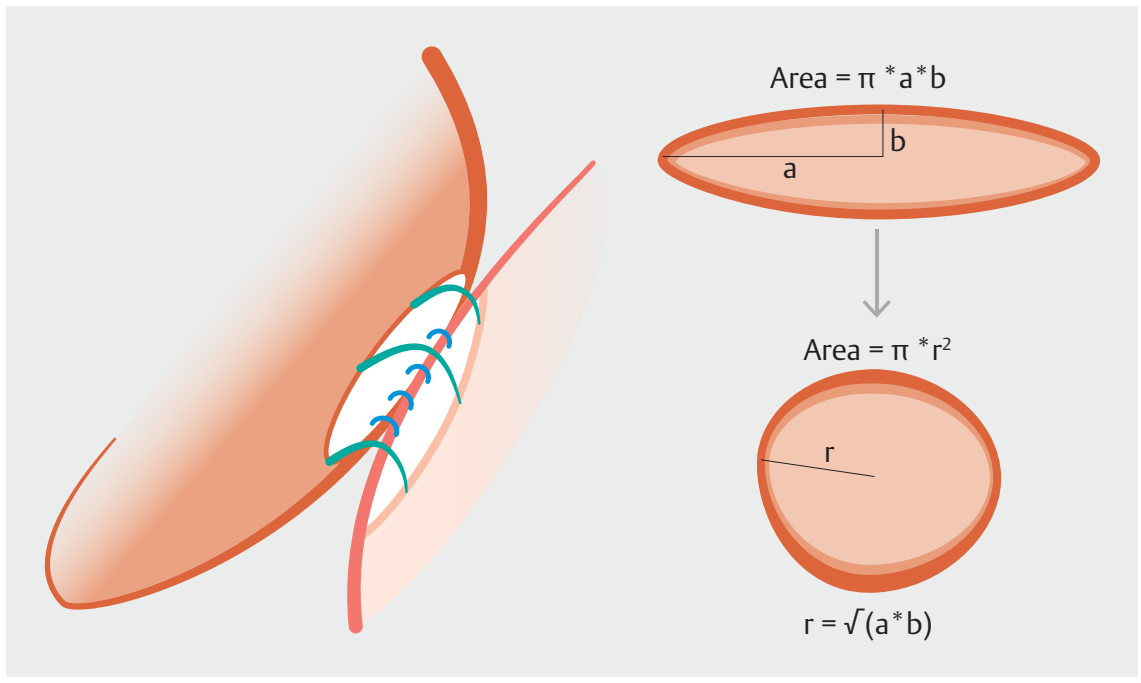

- Fig. 5 Pictorial representation of a surgical gastrojejunostomy. Left) Procedure requires the approximation of stomach and jejunal loops, a linear incision of their walls, and a latero-lateral suturing of their inferior and superior margins. This creates an elliptic anastomosis, which turns round after maturation and scarring. As surface areas must remain unchanged, the diameter of the final circular anastomosis will be inferior to the surgically made linear cut. Because the area of an ellipse is $\pi^{*} a^{*} b$ (the 2 semi-axes of the ellipse) and the area of a circle is $\pi^{*} r^{2}$ and the area cannot change during maturation, final radius of the circular anastomosis might be approximated to the square root of half the surgical linear incision.
[3] Rossi G, Petrone MC, Vanella G et al. EUSguided gastroenterostomy in a COVID-19infected patient with duodenal stenosing lymphoma (with videos). Endosc Ultrasound 2021; 10: 221-223. doi:10.4103/EUS-D-2000255

[4] Rodríguez JI, Kutscher M, Lemus M et al. Palliative gastrojejunostomy in unresectable cancer and gastric outlet obstruction: a retrospective cohort study. Ann R Coll Surg Engl 2021; 103: 197-202. doi:10.1308/ RCSANN.2020.7016

\section{Bibliography}

Endoscopy 2022; 54: E449-E451

DOI 10.1055/a-1562-1274

ISSN 0013-726X

published online 17.9.2021

(c) 2021. Thieme. All rights reserved.

Georg Thieme Verlag KG, Rüdigerstraße 14, 70469 Stuttgart, Germany

\section{ENDOSCOPY E-VIDEOS}

https://eref.thieme.de/e-videos

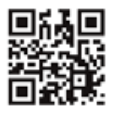

Endoscopy E-Videos is an open access online section, reporting on interesting cases and new techniques in gastroenterological endoscopy. All papers include a high quality video and all contributions are freely accessible online. Processing charges apply (currently EUR 375), discounts and wavers acc. to HINARI are available.

This section has its own submission website at https://mc.manuscriptcentral.com/e-videos 